\title{
INFLAMMATORY EVENTS AND OXIDANT PRODUCTION IN THE DIAPHRAGM, GASTROCNEMIUS, AND BLOOD OF RATS EXPOSED TO CHRONIC INTERMITTENT HYPOXIA: THERAPEUTIC STRATEGIES
}

\begin{tabular}{|r|l|}
\hline Journal: & Journal of Cellular Physiology \\
\hline Manuscript ID & JCP-16-0175.R1 \\
\hline Wiley - Manuscript type: & Original Research Article \\
\hline Complete List of Authors: & $\begin{array}{l}\text { Dominguez-Alvarez, Marisol; IMIM-Hospital del Mar, Universitat Pompeu } \\
\text { Fabra, Barcelona Biomedical Research Park (PRBB), Respiratory Medicine } \\
\text { Gea, Joaquim; IMIM-Hospital del Mar, Universitat Pompeu Fabra, Barcelona } \\
\text { Biomedical Research Park (PRBB), Respiratory Medicine } \\
\text { Barreiro, Esther; IMIM-Hospital del Mar, Universitat Pompeu Fabra, } \\
\text { Barcelona Biomedical Research Park (PRBB), CIBERES, 1Respiratory } \\
\text { Medicine-Muscle Wasting and Cachexia in Chronic Respiratory Diseases and } \\
\text { Lung Cancer, Health and Experimental Sciences Department (CEXS) }\end{array}$ \\
\hline Key Words: & $\begin{array}{l}\text { - experimental model of chronic intermittent hypoxia, - respiratory and } \\
\text { limb muscles, - inflammatory cytokines, - muscle inflammatory cells and } \\
\text { internal nuclei, superoxide anion production }\end{array}$ \\
\hline
\end{tabular}

\section{SCHOLARONE ${ }^{\text {M }}$}

Manuscripts 
Muscle and systemic hypoxia-induced inflammation and ROS

INFLAMMATORY EVENTS AND OXIDANT PRODUCTION IN THE DIAPHRAGM, GASTROCNEMIUS, AND BLOOD OF RATS EXPOSED TO CHRONIC INTERMITTENT HYPOXIA: THERAPEUTIC STRATEGIES

Marisol Domínguez-Álvarez ${ }^{1,2}$, Joaquim Gea $^{1,2}$, Esther Barreiro $^{1,2}$

${ }^{1}$ Respiratory Medicine-Muscle Wasting and Cachexia in Chronic Respiratory Diseases and Lung Cancer, IMIM-Hospital del Mar, Health and Experimental Sciences Department (CEXS), Universitat Pompeu Fabra, Universitat Autònoma de Barcelona, Barcelona Biomedical Research Park (PRBB), Dr. Aiguader, 88, Barcelona, E-08003 Spain.

${ }^{2}$ Centro de Investigación en Red de Enfermedades Respiratorias (CIBERES), Instituto de Salud Carlos III (ISCIII), Barcelona, Spain.

Corresponding author: Dr. Esther Barreiro, Pulmonology Department and Muscle and Respiratory System Research Unit, IMIM-Hospital del Mar, PRBB, Dr. Aiguader, 88, E08003 Barcelona, Spain, Telephone: +34 93316 0385, Fax: +34 93316 0410, e-mail: ebarreiro@imim.es.

RUNNING HEAD: Muscle and systemic hypoxia-induced inflammation and ROS

\section{WORD COUNT: 5,868}

\section{KEY WORDS:}

- experimental model of chronic intermittent hypoxia

- respiratory and limb muscles

- inflammatory cytokines

- ROS production

- muscle inflammatory cells and internal nuclei

- Number of text figures: 6

- Number of tables: 3

- Grant information: 
- Contract grant sponsor: Instituto de Salud Carlos III: CIBERES; FIS 11/02029 (FEDER); FIS 14/00713 (FEDER); SAF-2011-26908; SAF-2014-54371-R; Dr. Marisol Dominguez-Alvarez was a recipient of "Ayudas para la Investigación del Programa Río Hortega", Instituto de Salud Carlos III (Spain).

John Wiley \& Sons, Inc. 


\section{ABSTRACT}

We hypothesized that inflammatory events and reactive oxygen species (ROS) production may be differentially expressed in respiratory and limb muscles, and blood of a chronic intermittent hypoxia (CIH) experimental model and that antioxidants and TNF-alpha blockade may influence those events. In blood, diaphragm, and gastrocnemius of rats non-invasively exposed to $\mathrm{CIH}$ (10\% hypoxia, two hours/day, 14 consecutive days) with/without concomitant treatment with either anti-TNF-alpha antibody (infliximab) or N-acetyl cysteine (NAC), inflammatory cytokines, superoxide anion production, muscle structural abnormalities, and fiber-type composition were assessed. Compared to non-exposed controls, in CIH-exposed rats, body weight gain was reduced, TNF-alpha, IL-1beta, IL-6, and interferon-gamma levels were increased in diaphragm, TNF-alpha and IL-1 beta plasma levels were greater, systemic and muscle superoxide anion production was higher, diaphragm and gastrocnemius inflammatory cells and internal nuclei were higher, and muscle fiber-type and morphometry remained unmodified. CIH rats treated with infliximab further increased TNFalpha, IL-1beta, IL-6, and interferon-gamma diaphragm levels, whereas NAC induced a reduction only in TNF-alpha and IL-1beta levels in diaphragm and plasma. Infliximab and NAC elicited a significant decline in superoxide anion production in diaphragm, gastrocnemius, and plasma, while inducing a further increase in inflammatory cells and internal nuclei in both muscles. Proinflammatory cytokines are differentially expressed in respiratory and limb muscles and plasma of $\mathrm{CIH}$-exposed rats, while superoxide anion production increased in both muscle types and blood. Infliximab and NAC exerted different effects. These findings may help understand the biology underlying $\mathrm{CIH}$ in skeletal muscles and blood of patients with chronic respiratory diseases. Word count: 249 


\section{INTRODUCTION}

Patients with chronic respiratory diseases such as obstructive sleep apnea syndrome (OSAS) (Martinez et al, 2015) and patients with chronic obstructive pulmonary disease (COPD) during exercise may experience periods of chronic intermittent hypoxia (CIH) (GarciaTalavera et al, 2015). Interestingly, in OSAS recurrent episodes of complete or partial collapse of the upper airway during sleep induce apnea or hypopnea, respectively, with recurrent episodes of intermittent hypoxia (IH) during the night (Eckert et al, 2009; Garvey et al, 2009;Martinez-Garcia et al, 2015), which may lead to the development of systemic oxidative stress, as shown in several investigations (Lavie, 2008; Lavie et al, 2010;Lavie, 2012).

Importantly, similar features were demonstrated in experimental models of $\mathrm{CIH}$ such as increased vascular production of reactive oxygen species (ROS) (Troncoso Brindeiro et al, 2007), myocardial dysfunction with increased oxidative stress levels (Chen et al, 2005), increased platelet reactivity (Dunleavy et al, 2005), prolonged inflammatory response in the cerebral microcirculation (Altay et al, 2004), and increased atherosclerotic plaques (GautierVeyret et al, 2013). In chronic respiratory conditions (Barreiro et al, 2015;Maltais et al, 2014), skeletal muscles may also be affected by CIH. As such, the diaphragm of rodents exposed to hypoxia showed increased fatigue and reduced recovery time (McGuire et al, 2003), a slowto-fast muscle fiber transition (Shortt et al, 2013), and increased production of ROS that mediated diaphragm contractile failure (Shortt et al, 2014). Inflammatory parameters were also abundantly expressed in the larynx and soft palate tissues of rats in an experimental model of OSAS (Almendros et al, 2008). Moreover, a rise in oxidative stress and modifications in muscle structure were also shown in the external intercostal muscles (predominantly inspiratory muscles) of patients with OSAS, who concomitantly exhibited a reduction in respiratory muscle endurance (Barreiro et al, 2007). 
Recently, studies exploring the effects of chronic hypoxia on limb muscles in animal models have been conducted. For instance, hypoxia-induced atrophy was less prominent in oxidative than glycolytic limb muscles of mice exposed to chronic severe hypoxia (de Theije et al, 2015; Slot et al, 2016). Interestingly, in these animals, loss of muscle and fat tissues together with poor oxidative metabolism were observed along with a rise in proteolytic and autophagy markers following a three-week period of exposure to severe hypoxia $(8 \%)$. Importantly, short-term severe IH ( $6 \%$, four days) induced a differential expression profile of mechanisms involved in muscle atrophy in the diaphragm and tibialis anterior in another mouse model (Giordano et al, 2015). In this study, significant atrophy and increased lipid metabolism and autophagy markers were seen in the respiratory muscle, leading to the concept that those events may render the diaphragm more prone to fatigue (Giordano et al, 2015). Hence, these findings may have implications in patients with acute respiratory and cardiac conditions.

As most of the studies published so far have focused on models of relatively long or very short exposures to $\mathrm{CIH}$ of certain severity (Almendros et al, 2008; de Theije et al, 2015;McGuire et al, 2003; Shortt et al, 2013; Shortt et al, 2014; Slot et al, 2016; Giordano et al, 2015), elucidation of whether a short and less severe exposure to CIH may also modify the muscle phenotype and the inflammatory pattern is also of scientific interest. On this basis, we hypothesized that several inflammatory events and ROS production may be differentially expressed in the diaphragm and a limb muscle of similar fiber type composition and in blood of an experimental model (rats) of $\mathrm{CIH}$. Additionally, we also hypothesized that concomitant treatment with either anti-tumor necrosis factor (TNF)-alpha antibody or the antioxidant Nacetyl cysteine may attenuate the effects induced by $\mathrm{CIH}$ in the study muscles and blood compartment of the exposed animals. Accordingly, the study objectives were to explore: 1) levels of inflammatory cytokines, 2) superoxide anion production, 3) muscle structural abnormalities, and 4) fiber type composition in the diaphragm, gastrocnemius, and blood of 
rats exposed to a non-invasive model of moderate $\mathrm{CIH}$ (10\% hypoxia) for 14 consecutive days (including the weekend), with and without concomitant treatment with either anti-TNFalpha antibody or NAC.

\section{METHODS}

\section{Animal experiments and study design}

Animals. Pathogen-free male Wistar rats (Harlan, Horst, Netherlands) of identical age (8 weeks, 250-300 g) at baseline were used in the investigation. Food and water was supplied $a d$ libitum during the study period. In all rats, total body weight was determined by the same investigators twice: at baseline and at the end of the two-week study period.

Pharmacological agents. NAC powder (kindly provided by Dr. A. Esteras, Zambon SA, Barcelona, Spain) was dissolved in distilled water and prepared in order to obtain a final dose of $3 \mathrm{mmol} / \mathrm{kg}$. In the treated rats, NAC was administered daily using a 14-mm gauge needle (oral gavage). The NAC dose was chosen on the basis of previous studies (Barreiro et al, 2005; Barreiro et al, 2006;Dominguez-Alvarez et al, 2014). The anti-TNF-alpha antibody Infliximab was administered intraperitoneally $(0.01 \mathrm{mg} / \mathrm{g}$ in $0.3 \mathrm{~mL}$ volume $)$ on days 1 and 7 of the study protocol, also following previous studies in which the same compound was administered to rats and mice (Dominguez-Alvarez et al, 2014;Grounds and Torrisi, 2004). Infliximab is a chimeric immunoglobin (IgG1) containing $25 \%$ mouse-derived amino acids, the variable region, which is linked to a human constant region by disulphide bonds. Infliximab is produced in cell cultures using Chinese hamster ovary cells (Ordas et al, 2012). After subcutaneous injection, infliximab is absorbed slowly, probably via lymphatic drainage, reaching maximum concentrations between 8-10 days after administration.

Experimental protocol. The animals were first placed, fully awake, in a polyurethane plethysmographic chamber, the anterior part of which contained a two-way threshold valve (Hans Rudolph, model 2200, Kansas City, MO, USA), whose inspiratory branch was 
connected to a Douglas's bag which was coupled to a gas source $\left(10 \% \mathrm{O}_{2}\right)$. All the animals were exposed to either $10 \% \mathrm{O}_{2}$ or room air for two hours/day for 14 consecutive days (including the weekends). Saturation of oxyhemoglobin (pulse oximeter), heart rate, expiratory fraction of $\mathrm{CO}_{2}$ (end-tidal $\mathrm{CO}_{2}$, to prevent rebreathing) were also monitored throughtout the entire study period. In the animals exposed to intermittent hypoxia, arterial blood gas analysis was conducted from the tail to verify that $\mathrm{PaO}_{2}$ levels were indeed below $60 \mathrm{mmHg}$ in all rats. All the animals were exposed to the same dietary conditions. The animals were given food and water ad libitum and no differences were observed between the groups.

Study groups and protocol. Thirty-two rats were studied in the investigation. All rats were randomly assigned to the different experimental groups ( $\mathrm{N}=8$ /group). The following groups were established in the study: 1) non-exposed control group, in which animals were not exposed to hypoxia, but lived in identical environmental conditions as the other groups (exposed to the chamber in a normal atmosphere for two consecutive hours/24 hours for 14 consecutive days), in order to control for stress-related factors, 2) CIH group, animals were exposed to $10 \% \mathrm{O}_{2}$ for two consecutive hours/24 hours for 14 consecutive days, 3) anti-TNFalpha-hypoxia group, in which animals exposed to identical hypoxia conditions received concomitant treatment with anti-TNF-alpha antibody/7days for 14 consecutive days (two intraperitoneal injections in total on days 0 and 7), and 4) NAC-hypoxia group in which animals concomitantly received treatment with $3 \mathrm{mmol} / \mathrm{kg} \mathrm{NAC} / 24 \mathrm{~h}$ (oral gavage) for 14 consecutive days. None of the animals died during the study period. On day 14 of the study protocol, after finishing the hypoxia or the control exposures, animals from the different experimental groups were sacrificed. Animals were exsanguinated and blood was collected from the cardiac cavities and stored at $-80^{\circ} \mathrm{C}$ until further use. The diaphragm and gastrocnemius were then quickly excised. All muscle samples were either immediately frozen in liquid nitrogen and subsequently stored at $-80^{\circ} \mathrm{C}$ until further use (molecular analyses) or 
immersed in an alcohol-formol bath for two hours to be thereafter embedded in paraffin (tissue analyses).

\section{Ethical approval}

All animal experiments were conducted in the animal facilities at Parc de Recerca Biomèdica de Barcelona (PRBB, Spain). This controlled study was designed in accordance with both the ethical standards on animal experimentation (EU 609/86 CEE, Real Decreto 1201/05 BOE 252, Spain) at PRBB and the European Convention for the Protection of Vertebrate Animals Used for Experimental and Other Scientific Purposes (1986). The study conforms to the current international ethical guidelines for animal research. Ethical approval was obtained by the Animal Research Committee at PRBB (JGG-13-1540).

\section{Muscle biology analyses}

Detection of superoxide anion radicals in muscle compartments. The reagents used in these experiments were all purchased from Sigma (Sigma, Saint Louis, MO, USA). Frozen muscle specimens from diaphragm and gastrocnemius of all groups were fractionated into cytosolic, membrane, and mitochondria compartments following previous reports by some of us (Dominguez-Alvarez et al, 2014;Fermoselle et al, 2012;Marin-Corral et al, 2009). The entire procedure was done at $4^{\circ} \mathrm{C}$ (on ice). In brief, frozen muscle samples were homogenized in 6 v/w ice-cooled homogenization buffer A [50 mM 4-(2-hydroxyethyl)-1piperazineethanesulfonic acid (HEPES), $0.1 \mathrm{mM}$ Dithiothreitol (DTT), $2 \mu \mathrm{g} / \mathrm{ml}$ leupeptin, 100 $\mu \mathrm{g} / \mathrm{ml}$ phenylmethylsulfonyl fluoride (PMSF), $2 \mu \mathrm{g} / \mathrm{ml}$ aprotinin, $1 \mathrm{mg} / 100 \mathrm{ml}$ pepstatin A, $5 \%$ glycerol, $\mathrm{pH} 7.4]$. Samples were then centrifuged at $1,000 \mathrm{~g}$ for 10 minutes. The pellet (P1) was discarded, whereas the supernatant (S1) was designated as crude homogenates. These homogenates were then centrifuged at $12,000 \mathrm{~g}$ for 20 minutes to yield supernatant (S2) and pellet (P2). Pellet (P2) was then re-suspended in buffer B [10 mM tris-maleate, 0.1 $\mathrm{mM}$ ethylenediaminetetraacetic acid (EDTA), and $135 \mathrm{mM} \mathrm{KCl]}$ and then centrifuged at $12,000 \mathrm{~g}$ for 20 minutes to yield S3 and P3. The resulting pellet (P3) was re-suspended in 
buffer A and designated as the mitochondrial fraction. Both S2 and S3 fractions were pooled and were used to separate the membrane and cytosolic fractions by centrifugation for one hour at 100,000 g. The resulting supernatant (S4) was designated as the cytosolic fraction, whereas the pellet (P4) was re-suspended in buffer C (10 mM HEPES, $300 \mathrm{mM}$ sucrose, $\mathrm{pH}$ 7.2), treated for one hour with $600 \mathrm{mM} \mathrm{KCl}$ and then centrifuged again at 100,000 $\mathrm{g}$ for another hour. Pellet was re-suspended in buffer A and designated as the membrane fraction.

After a 10-minute preincubation period of the samples at $37^{\circ} \mathrm{C}$, baseline measurements of the different muscle fractions were made in a luminometer (Lumat LB 9507, Berthold Technologies GmbH, Bad Wildbad, Germany) by adding $250 \mu \mathrm{M}$ lucigenin for five minutes. Moreover, in order to confirm the contribution to superoxide anion production of each muscle compartment, additional groups of muscle fractions were further incubated for 20 minutes at $37^{\circ} \mathrm{C}$ with $250 \mu \mathrm{M}$ rotenone (inhibitor of mitochondrial complex I activity) in the mitochondrial fraction, $200 \mu \mathrm{M}$ apocynin (specific inhibitor of NADPH oxidase) in the membrane fraction, and $300 \mu \mathrm{M}$ oxypurinol (inhibitor of xanthine oxidase) in the cytosolic fraction. Incubation with the corresponding inhibitors significantly reduced the luminometer output in each muscle fraction of all the samples.

The specificity of the method was tested by preincubation of a group of muscle fractions (mitochondria, membrane, and cytosol) from control and hypoxia rats with and without the pharmacological treatments with $0.33 \mathrm{U} / \mathrm{ml}$ superoxide dismutase (SOD) for twenty minutes at $37^{\circ} \mathrm{C}$, to which $250 \mu \mathrm{M}$ lucigenin was added and the luminometer output was recorded for five minutes. Preincubation of the samples with superoxide dismutase significantly diminished the production of superoxide anion in all the muscle fractions compared to baseline measurements. Intra-assay coefficients of variation for these methodologies ranged from $2 \%$ to $4 \%$, while inter-assay coefficients of variation ranged from $7 \%$ to $10 \%$ 
Detection of superoxide anion radicals in blood. The reagents employed in these methodologies were all purchased from Sigma. In order to quantify superoxide anion production, lucigenin-derived chemiluminescence (LDCL) signals were determined in all blood (serum) samples using a luminometer (Lumat LB 9507, Berthold Technologies GmbH, Bad Wildbad, Germany) as formerly described (Dominguez-Alvarez et al, 2014;Fermoselle et al, 2012;Marin-Corral et al, 2009). Briefly, $50 \mu \mathrm{L}$ of each serum sample was poured in a tube containing $950 \mu \mathrm{L}$ Krebs-HEPES buffer. The mixture was incubated for 10 minutes at $37^{\circ} \mathrm{C}$ in a water bath. Lucigenin $(0.1 \mathrm{mM})$ was immediately added after the 10 -minute incubation period and the tubes were subsequently placed in the luminometer. The luminometer output was read during 10 minutes in all the samples. LDCL signals were measured in the presence of lucigenin alone (baseline levels) and in the presence of $0.5 \mathrm{U} / \mathrm{mL}$ SOD.

Cytokine Enzyme-linked Immunosorbent Assay (ELISA). Protein expression of the cytokines TNF-alpha, IL-1beta, IL-6, and interferon-gamma was quantified in the diaphragm, gastrocnemius and plasma of all the experimental groups using specific-species sandwich ELISA kits (Bender Medsystems GmbH, Vienna, Austria) following similar previously published methodologies (Barreiro et al, 2008;Barreiro et al, 2010;Chacon-Cabrera et al, 2014a;Dominguez-Alvarez et al, 2014). Frozen muscle sample specimens were homogenized and protein concentration calculated as described above. For all the sample specimens equal amounts of total protein from muscle homogenates were always loaded in triplicates $(15 \mu \mathrm{g}$ in $200 \mu \mathrm{L}$ total volume each singlet for all the triplicates of all the study samples) onto the ELISA plates. All samples were incubated with the specific primary antibodies and were always run together in each assay. Before commencing the assay, samples and reagents were equilibrated to room temperature. A standard curve was always run with each assay run. Standards $(200 \mu \mathrm{L})$ and the protocols were prepared according to the manufacturer's instructions for each cytokine. Absorbances were read at $450 \mathrm{~nm}$ using as a reference filter that of $655 \mathrm{~nm}$. Intra-assay coefficients of variation for the different cytokines and studies 
ranged from $4.5 \%$ to $10 \%$. Inter-assay coefficients of variation for the same cytokines ranged from $8 \%$ to $12 \%$.

Muscle fiber counts and morphometry. On 3-micrometer muscle paraffin-embedded sections of both diaphragms and gastrocnemius muscles from all animal groups, myosin heavy chain (MyHC)-I and-II isoforms, were identified using anti-MyHC-I (clone NOQ7.5.4D, Sigma, Saint Louis, MO, USA), anti-MyHC-II (clone MY-32, Sigma, Saint Louis, MO), as published elsewhere (Barreiro et al, 2008;Barreiro et al, 2010;Chacon-Cabrera et al, 2014a;DominguezAlvarez et al, 2014). The cross-sectional area, mean least diameter, and proportions of type I and type II fibers were assessed using a light microscope (Olympus, Series BX50F3, Olympus Optical Co., Hamburg, Germany) coupled with an image-digitizing camera (Pixera Studio, version 1.0.4, Pixera Corporation, Los Gatos, CA, USA) and a morphometry program (NIH Image, version 1.60, Scion Corporation, Frederick, MD, USA). In order to explore muscle fiber types and sizes at least 100 fibers were measured and counted in each muscle specimen from all animals.

Muscle structure abnormalities. The area fractions of normal and abnormal muscle (abnormal cellular events taking place in the muscle fibers) was evaluated on three-micrometer paraffinembedded sections of diaphragms and gastrocnemius of all rats following similar methodologies published elsewhere (Chacon-Cabrera et al, 2014a;Dominguez-Alvarez et al, 2014). Briefly, normal and abnormal tissue was quantified using computer-assisted point counting in all the muscle sections, previously stained with hematoxylin-eosin. A grid of 63 point-intercepts ( $7 \times 9$ rectangular pattern), built by means of the software Imaging Cell-B (Olympus Corporation), was superimposed onto the image of the muscle cross section at a magnification of 400x under a light microscope (Olympus BX 61, Olympus Corporation) using an image digitizing camera (Olympus DP 71, Olympus Corporation). Thirty images were measured in each muscle sample of all animal groups. Each point-intercept (field) was assigned to a specific category and entered into the software. A total of 1,890 fields were 
analyzed in each muscle sample. Categories for point counting were defined as follows: 1) normal muscle, 2) internal nuclei, 3) inflammatory cells, 4) lipofuscin, 5) abnormal viable, 6) inflamed/necrotic, 7) vessel, and 0) no count. The area fraction for each category was defined as the proportion of points that fell on each of these traits relative to the total number of points superimposed on all viable fields (all features except for categories 0 and 7) of each cross section. The area fraction of normal muscle was equivalent to the proportion of points falling in category 1 , while the area fraction of abnormal muscle was determined by calculating the proportion of points in categories $2,3,4,5$, and 6 .

\section{Statistical analysis}

Results are expressed as mean and standard deviation. Physiological variables (body weight) were analyzed as follows: 1) comparisons were made between baseline, and post-hypoxia period in each experimental group of rats; 2) comparisons were made between non-exposed control group and any of the experimental hypoxia groups; and 3) comparisons were made between rats exposed to hypoxia treated with either NAC or anti-TNF-alpha antibody and those exposed to hypoxia-only (control group) for this set of comparisons.

All biological variables (from muscles and blood) were analyzed at the end of the study period and comparisons were made as follows: 1) between the non-exposed controls and any of the experimental hypoxia groups, and 2) between rats exposed to hypoxia treated with either NAC or anti-TNF-alpha antibody and animals exposed to hypoxia-only, which was the control group in this type of analysis.

Comparisons of physiological variables between post-hypoxia and baseline timepoints were explored using parametric paired T-test. At the end of the study period, differences in both physiological and biological variables (muscle and blood compartments) between the different study groups were assesed using one-way analysis of variance, and Dunnett's post-hoc analysis was used to adjust for multiple comparisons. The sample size chosen was based on previous studies, where very similar approaches were employed 
(Barreiro et al, 2005; Barreiro et al, 2006; Chacon-Cabrera et al, 2014a;Chacon-Cabrera et al, 2014b;Chacon-Cabrera et al, 2015;Dominguez-Alvarez et al, 2014) and on assumptions of $80 \%$ power to detect an improvement of more than $20 \%$ in measured outcomes at a level of significance of $P \leq 0.05$.

\section{RESULTS}

\section{Physiological characteristics}

As shown in Table 1 and Figure 1, only the non-exposed control group gained a significant amount of body weight after the two-week study period compared to baseline. At the end of the two-week study period, the gain in total body weight was significantly smaller in all hypoxia groups than in the non-exposed control animals (Table 1 and Figure 1). Treatment with either NAC or anti-TNF-alpha antibody did not significantly improve body weight gain in the rats at the end of the study period (Table 1 and Figure 1).

\section{Systemic and muscle inflammatory markers}

After the two-week study period, protein levels of TNF-alpha were significantly greater in the diaphragm of hypoxia rats than in the non-exposed controls (Figure 2, top panel). Interestingly, treatment of hypoxia rats with anti-TNF-alpha antibody elicited a further increase in protein levels of the cytokine TNF-alpha in their diaphragms compared to hypoxia-only rodents and the non-exposed controls (Figure 2, top panel). Hypoxia animals treated with NAC experienced a significant decline in TNF-alpha levels in their diaphragm compared to hypoxia-only rodents (Figure 2, top panel). In the gastrocnemius, TNF-alpha protein levels did not differ among any of the study groups (Figure 2, medium panel). In plasma, TNF-alpha protein levels showed a significant increase in CIH-exposed rats compared to non-exposed controls (Figure 2, bottom panel). Treatment with anti-TNF-alpha antibody did not significantly modify TNF-alpha levels in plasma compared to either hypoxia rats or the non-exposed controls (Figure 2, bottom panel). Concomitant treatment of these 
animals with NAC elicited a significant decline in plasma TNF-alpha levels compared to hypoxia-only rats but not to the non-exposed controls.

Protein levels of IL-1beta were significantly greater in the diaphragm of hypoxia rats than in the non-exposed controls (Figure 3, top panel). Protein levels of such a proinflammatory cytokine were further increased in the diaphragm of anti-TNF-alphahypoxia rodents compared to hypoxia-only and non-exposed control rats (Figure 3, top panel). Nonetheless, treatment with NAC elicited a decrease in diaphragm IL-1beta levels compared to hypoxia-only animals, whereas a significant increase was observed compared to the non-exposed controls (Figure 3, top panel). Protein levels of IL-1beta did not significantly differ in the limb muscle among any of the study groups (Figure 3, medium panel). In plasma, IL-1 beta protein levels showed a significant increase in the hypoxia group compared to nonexposed controls (Figure 3, bottom panel). Plasma levels of such a cytokine exhibited a significant decline in both anti-TNF-alpha-hypoxia and NAC-hypoxia groups of rodents compared to hypoxia-only animals, with similar levels to those detected in the non-exposed controls (Figure 3, bottom panel).

Protein levels of IL-6 were significantly increased in the diaphragm of hypoxia rats compared to the non-exposed controls (Figure 4, top panel). Moreover, IL-6 protein levels were even greater in the diaphragm of rats treated with the anti-TNF-alpha antibody than in hypoxia-only rats and non-exposed controls (Figure 4, top panel). Concomitant treatment with NAC only elicited a significant increase in IL-6 levels compared to the non-exposed controls, but not to hypoxia-only rats (Figure 4, top panel). In the limb muscle and blood compartment, no significant differences were observed in IL-6 protein levels among the study groups (Figure 4, medium and bottom panels).

Protein levels of interferon-gamma were increased in the diaphragm of the hypoxia rats compared to the non-exposed controls (Figure 5, top panel). In the same muscle, no significant differences were observed between NAC-hypoxia and hypoxia only groups, while 
treatment with anti-TNF-alpha antibody induced a further increase in TNF-alpha levels compared to hypoxia-only rats and the non-exposed controls (Figure 5, top panel). Protein levels of interferon-gamma did not significantly differ in either gastrocnemius muscle or plasma compartment among any of the study groups (Figure 5, medium and bottom panels, respectively).

\section{Systemic and muscle oxidant production}

Generation of superoxide anion was greater within the mitochondrial and membrane compartments in the diaphragm of hypoxia rats than in the non-exposed controls (Figure 6A, top and medium panels, respectively). Interestingly, in the diaphragm of both anti-TNF-alphahypoxia and NAC-hypoxia rats a significant decline in superoxide anion levels was observed within the mitochondrial and membrane compartments compared to hypoxia-only rodents, while levels were similar to those detected in the non-exposed controls (Figure 6A, top and medium panel, respectively). In the cytosolic fraction of the diaphragm, superoxide anion levels were very low and did not significantly differ among the study groups (Figure 6A, bottom panel). In the gastrocnemius of hypoxia rats, levels of superoxide anion were higher within the mitochondrial compartment compared to those detected in the non-exposed control group (Figure 6B, top panel). As in the diaphragm, in the gastrocnemius of anti-TNF-alphahypoxia and NAC-hypoxia rats, a significant reduction in superoxide anion levels was detected within the mitochondrial compartment compared to hypoxia-only rodents (Figure 6B, top panel). Nevertheless, no differences in superoxide anion production were detected among the study groups within the membrane or cytosolic fractions in the gastrocnemius muscle (Figure 6B, medium and bottom panels, respectively). As in both study muscles, plasma levels of superoxide anion production were greater in hypoxia group of rats than in the non-exposed controls (Figure $6 \mathrm{C}$ ). Interestingly, in anti-TNF-alpha-hypoxia and NAChypoxia groups, superoxide anion levels were significantly reduced in plasma compared to 
hypoxia-only group, and were similar to those detected in the non-exposed controls (Figure $6 \mathrm{C})$.

\section{Muscle structure}

In diaphragm and gastrocnemius muscles of all groups of rats no significant differences were observed in either muscle fiber-type composition or sizes (Table 2).

Proportions of total structural abnormalities were increased within the diaphragm and gastrocnemius muscles of hypoxia rats compared to the non-exposed controls (Table 3). Interestingly, treatment of hypoxia rats with either anti-TNF-alpha antibody or NAC elicited a further increase in structural abnormalities in their diaphragms and gastrocnemius compared to hypoxia-only rodents and the non-exposed controls, as a result of a significant rise in the number of both inflammatory cells and internal nuclei (Table 3).

\section{DISCUSSION}

In the study, the hypothesis has been confirmed to a great extent. A differential pattern of inflammatory events was observed in the blood and the main respiratory muscle compared to the gastrocnemius in rats exposed to $\mathrm{CIH}$ for two consecutive weeks. Moreover, concomitant treatment with either the antioxidant or anti-TNF-alpha antibody elicited relevant modifications in the levels of ROS production and the inflammatory parameters analyzed in the study that are discussed below. Interestingly, $\mathrm{CIH}$ reduced body weight gain compared to non-exposed controls, and treatment with either infliximab or NAC did not modify those effects on whole body weight in the animals. These findings are also discussed in the next paragraphs.

Profile of inflammatory events in respiratory and limb muscles and blood: effects of infliximab and NAC

Inflammatory cytokines were differentially expressed in the diaphragm, gastrocnemius and blood study compartments of the rats exposed to $\mathrm{CIH}$. Moreover, the cytokine response to 
each of the pharmacological agents also differed among the three compartments. As such, levels of TNF-alpha were significantly greater in the diaphragm and blood of the rats chronically exposed to $\mathrm{CIH}$ than in non-exposed animals. Importantly, concomitant treatment with anti-TNF-alpha antibody of the hypoxia rats induced a further increase in TNF-alpha levels only in the diaphragm, while inducing no significant differences in the systemic levels of this cytokine. These findings are in line with previous results (Dominguez-Alvarez et al, 2014), in which the antibody infliximab elicited a larger increase in TNF-alpha protein levels in the diaphragm of rats exposed to high-inspiratory loads. We concluded that infliximab most likely blocked systemic TNF-alpha but not that synthesized within the fibers of the respiratory muscle, whose levels were even greater after treatment with the pharmacological agent in response to both high-inspiratory loading (Dominguez-Alvarez et al, 2014) and hypoxia in the current study.

Protein levels of IL-1beta were significantly larger in the diaphragm and blood of the $\mathrm{CIH}$-exposed rats than in the non-exposed controls. Importantly, concomitant treatment with infliximab also induced a further increase in IL-1beta in the respiratory muscle, while a significant decline was detected in the blood compartment of the same animals. In keeping with, a rise in the levels of the cytokines IL-6 and interferon-gamma was also observed in the diaphragm of the animals exposed to $\mathrm{CIH}$ compared to non-exposed rats, and concomitant treatment with the anti-TNF-alpha antibody induced a further increase in the levels of those cytokines in the respiratory muscle. Systemic levels of IL-6 and interferon-gamma, however, were not modified by infliximab in the $\mathrm{CIH}$-exposed animals. These results suggest that the effects of the anti-TNF-alpha antibody differ between blood and the respiratory muscle compartments in this model of $\mathrm{CIH}$ of relatively short duration (15 days). Taken together, these findings indicate that infliximab may trigger a process of muscle regeneration (as demonstrated by the increased internal nuclei counts) in response to moderate $\mathrm{CIH}$ in the diaphragm characterized by a significantly greater increase in several cytokines such as TNF- 
alpha, IL-1 beta, IL-6, and interferon-gamma than in hypoxia rats without the treatment. Nonetheless, systemic levels of the proinflammatory cytokines were significantly decreased (IL-1 beta) in response to infliximab or were not modified in the CIH-exposed rats. As abovementioned, all these results suggest again that the antibody rather blocks the production of systemic proinflammatory cytokines than those synthesized within the muscle fibers. Indeed, similar findings were found in a previous study, in which infliximab also elicited a significant reduction in blood levels of several cytokines in animals exposed to high-intensity inspiratory loads, while inducing an increase of proinflammatory cytokines in the loaded muscle (diaphragm) (Dominguez-Alvarez et al, 2014).

Importantly, no significant differences in cytokine levels were observed in the limb muscle among the study groups, implying that hypoxia or any of the pharmacological agents did not modify the cytokine profile in the gastrocnemius. The activity of the respiratory muscle, as opposed to the limb muscle, most likely accounts for the differential expression profile of cytokines between the two muscles. In fact, previous investigations have also demonstrated a rise in inflammatory cytokines only in the respiratory muscle following resistive breathing (Sigala et al, 2012;Vassilakopoulos et al, 2004) and high-inspiratory threshold loading (Dominguez-Alvarez et al, 2014) in rats.

The antioxidant NAC elicited a significant decrease in TNF-alpha levels in the diaphragm and blood of the rats exposed to $\mathrm{CIH}$ compared to non-treated hypoxia animals. Similarly, levels of IL-1 beta were also significantly lower in the respiratory muscle and blood of rodents receiving treatment with NAC than in non-treated hypoxia rats. NAC did not induce any significant reduction in levels of IL-6 or interferon-gamma in any of the study muscles or blood compartment of $\mathrm{CIH}$-exposed rats compared to non-treated hypoxia animals. Collectively, these results imply that the antioxidant effects of NAC may be mediated through an antiinflammatory mechanism (TNF-alpha and IL-1 beta) both in the respiratory muscle and blood. It is likely that muscle activity influences to a great extent the 
profile of proinflammatory cytokines such as TNF-alpha and IL-1 beta in the diaphragm of the CIH-exposed rats in response to the antioxidant treatment. In fact, recent observations from experimental models have also demonstrated that NAC induced its beneficial effects on several tissues and muscles through a TNF-alpha dependent mechanism (de Senzi Moraes et al, 2013; Lin et al, 2015; Moraes et al, 2015).

The findings encountered in this study are also in agreement with those previously shown, in which resistive breathing induced a rise in several proinflammatory cytokines in the rat diaphragm mediated by major signaling pathways such as nuclear factor (NF)-kB and mitogen-activated protein kinases (MAPKs), and pretreatment of the animals with NAC completely blunted the production of those inflammatory cytokines (Sigala et al, 2011). The authors concluded that oxidative stress was a main trigger for the increased activity-induced upregulation of cytokines seen in the diaphragm (Sigala et al, 2011). Other studies have also shown that oxidative stress may play a significant role in the inflammatory response induced by increased muscle activity. In this regard, treatment with NAC was shown to attenuate the activity-induced increase in systemic levels of proinflammatory cytokines in healthy humans following general exercise (Vassilakopoulos et al, 2003), strenuous resistive breathing (Vassilakopoulos et al, 2002), and in monocytes (Toumpanakis et al, 2009). Finally, it should be mentioned that NAC did not elicit any significant change in the levels of the study cytokines in the limb muscle. As abovementioned activity of the target muscle determines the expression profile of cytokines.

Oxidative stress profile in respiratory and limb muscles and blood compartment: effects of infliximab and NAC

A significant rise in superoxide anion production levels was observed in the mitochondrial and membrane fractions of the diaphragm in rats exposed to CIH. Moreover, a significant increase in superoxide anion levels was also observed in the mitochondrial fraction of the limb muscle in the same animals. These findings suggest that mitochondria are major targets 
of hypoxia-induced oxidative stress as superoxide anion release from this specific compartment was observed in both types of muscles as also shown to occur in other conditions (Taivassalo and Hussain, 2016). Moreover, mitochondria was the most relevant contributor to oxidant production as levels were larger than in the other cell compartments and were, indeed, increased in both respiratory and limb muscles of the CIH-exposed animals. These results are in agreement with former investigations, in which increased oxidant production was also shown in the muscles of COPD patients with severe hypoxia (Koechlin et al, 2005), in diaphragm of rats exposed to high-inspiratory loading (Dominguez-Alvarez et al, 2014), and in both respiratory and limb muscles of mice exposed to very short IH (Giordano et al, 2015). Systemic levels of superoxide anion were also significantly greater in the animals exposed to $\mathrm{CIH}$ compared to non-exposed control rats. Collectively, these results imply that hypoxia induced systemic effects on ROS production as it enhanced oxidant production systemically and in both muscle types of the rats.

Interestingly, treatment with infliximab elicited a significant decline in systemic superoxide anion production, in both mitochondria and membrane compartments of the respiratory muscle, and in the mitochondrial fraction of the gastrocnemius in rats exposed to $\mathrm{CIH}$. As inflammatory cell counts were actually further increased in response to infliximab in both diaphragm and gastrocnemius, superoxide anion production levels were probably reduced as a result of a mechanism relying on the actual myofibers (mitochondrial and membrane-ROS producing enzymes) of the CIH-exposed animals treated with infliximab rather than to an inflammatory cell-dependent mechanism (de Senzi Moraes et al, 2013; Langen et al, 2002).

Importantly, levels of superoxide anion were significantly lower in the mitochondrial fraction of both diaphragm and gastrocnemius and in the membrane compartment of the former muscle in hypoxia rats treated with NAC than in hypoxia-only animals. Additionally, systemic levels of superoxide anion were also significantly reduced in the plasma of hypoxia 
rodents treated with the antioxidant compared to those without the treatment. In line with this, plasma and diaphragm superoxide anion production levels also decreased in rats exposed to inspiratory threshold loading that were concomitantly treated with NAC (Dominguez-Alvarez et al, 2014). As abovementioned treatment with NAC also elicited a significant reduction in systemic and muscle oxidative stress and inflammatory cytokine levels in several human studies (Toumpanakis et al, 2009; Vassilakopoulos et al, 2002; Vassilakopoulos et al, 2003) and animal models of resistive breathing (Sigala et al, 2011; Sigala et al, 2012). Collectively, these results suggest that NAC is a potent antioxidant that exerts its effects in all body compartments.

Similar structural abnormalities in respiratory and limb muscles and body weight: effects of infliximab and NAC

The proportions of structural abnormalities and those of internal nuclei and inflammatory cells were greater in both diaphragm and gastrocnemius of $\mathrm{CIH}$-exposed animals compared to non-exposed control animals. These findings imply that exposure to moderate levels of $\mathrm{CIH}$ induced a process of muscle regeneration in the rats. Furthermore, concomitant treatment with either infliximab or NAC elicited a significant rise in total muscle abnormalities characterized by an increase in the number of inflammatory cells and internal nuclei in both respiratory and limb muscles of rats exposed to $\mathrm{CIH}$ compared to non-treated hypoxia animals. These are very interesting findings, which are in line with previous studies in which chronic respiratory patients with hypoxia also exhibited a larger increase in inflammatory cell counts in the study muscles (Barreiro et al, 2010;Koechlin et al, 2005).

On the other hand, NAC and infliximab may have also triggered a repair process (increased internal nuclei counts) in response to hypoxia, which could be partly mediated through the rise in inflammatory cells observed in both study muscles. Interestingly, a decline in levels of several markers of oxidative stress and damage was also observed in myotubes of distrophic mice that had been treated with the antioxidant NAC (Moraes et al, 2015). 
Moreover, levels of muscle damage (necrosis) in the diaphragm also decreased in distrophic mice treated with NAC as a result of a decline in TNF-alpha and oxidative stress levels (de Senzi Moraes et al, 2013). All these findings indicate that NAC antioxidant effects alleviate muscle damage, while promoting muscle repair and regeneration of respiratory and limb muscles in several experimental models.

It should be underscored that fiber-type composition or morphometry were not modified by exposure to $\mathrm{CIH}$ in any study muscle or by the administration of either pharmacological agent. These results are not totally in agreement with those reported in previous investigations, in which a smaller size of fast-twitch fibers was demonstrated in patients with advanced COPD and cachexia (Fermoselle et al, 2012;Puig-Vilanova et al, 2015b;PuigVilanova et al, 2015a), in animals with cancer cachexia (Chacon-Cabrera et al, 2014a; Chacon-Cabrera et al, 2015), in mice exposed to chronic continuous severe hypoxia (de Theije et al, 2015; Slot et al, 2016), and in mice exposed to short IH (Giordano et al, 2015). Differences in the study models (intensity of hypoxia, duration, and continuous versus intermittent) may account for the discrepancies of the results observed in the different study models.

Finally, it is worth mentioning that $\mathrm{CIH}$ exposure induced a significantly lower body weight gain in the rats than that observed in the non-exposed animals. These findings are consistent with those previously reported in experimental models of chronic continuous severe hypoxia or CIH (de Theije et al, 2015;Giordano et al, 2015; Shortt et al, 2014;Slot et al, 2016). Several biological mechanisms may underlie the body weight loss induced by hypoxia. For instance, hypoxia was shown to inhibit protein synthesis (Preedy et al, 1985), to alter mitochondrial enzymes and muscle mass (Bigard et al, 1991), to induce an aerobic to anaerobic shift due to mitochondrial loss (Howald et al, 1990), to reduce mitochondrial protein synthesis (Kwast and Hand, 1996), and to increase leptin concentrations while reducing those of metabolic parameters (sugar and cholesterol) in experimental models (Ling 
et al, 2008). As the sizes of the fibers were not reduced in any of the study muscles, we propose that metabolic and mitochondrial alterations are probably the most relevant contributors to the decrease in body weight observed in the CIH-exposed rats. Inflammatory cytokines or ROS were probably not involved in the body weight loss of the hypoxia animals, since treatment with either infliximab or NAC did not significantly improve this parameter.

\section{Conclusions}

Proinflammatory cytokines are differentially expressed in the respiratory and limb muscles and plasma of rats exposed to non-invasive $\mathrm{CIH}$, while superoxide anion production was increased in both muscle types and blood. The anti-TNF-alpha antibody and NAC probably triggered muscle regeneration, which was characterized by a rise in inflammatory cell counts and internal nuclei in both diaphragm and gastrocnemius of rats exposed to $\mathrm{CIH}$, while no modifications in muscle morphometry or fiber type composition were observed among the study groups. Concomitant treatment of the hypoxia animals with infliximab induced a further increase in levels of proinflammatory cytokines in the diaphragm, whereas NAC elicited a significant decline in superoxide anion levels in all compartments. These findings may help understand the biology underlying $\mathrm{CIH}$ in skeletal muscles and blood of patients with chronic respiratory diseases. 


\section{LIST OF ABBREVIATIONS}

$\mathrm{CIH}$, chronic intermittent hypoxia; COPD, chronic obstructive pulmonary disease; DTT, Dithiothreitol; EDTA, Ethylenediaminetetraacetic acid; ELISA, enzyme-linked immunosorbent assay; HEPES, 4-(2-hydroxyethyl)-1-piperazineethanesulfonic acid; IL, interleukin; IgG, immunoglobin G; IH, intermittent hypoxia; LDCL, lucigenin-derived chemiluminescence; MyHC, myosin heavy chain; NAC, N-acetyl cysteine; OD, optical densities; OSA, obstructive sleep apnea, PMSF, phenylmethylsulfonyl fluoride; ROS, reactive oxygen species; SOD, superoxide dismutase; TNF, tumor necrosis factor. 


\section{ACKNOWLEDGMENTS}

The authors gratefully acknowledge Francisco Sanchez-Corredera, Anna Salazar-Degracia, Mònica Vila-Ubach, and Marina Sabaté-Brescó for their technical assistance with part of the animal and molecular biology experiments, and to Dr. Xavier Mateu for his help with the use of the pharmacological compounds.

Editorial support: None to declare. CONFLICT OF INTEREST

The authors declare no conflict of interest in relation to this study.

\section{AUTHOR CONTRIBUTIONS}

MDA has conducted the animal experiments, obtained the biological samples, has performed the in vivo physiological and molecular biology experiments, and has contributed to manuscript writing.

JG contributed to the study design, data analyses, organization of the study results, intellectual input, and manuscript writing.

EB has contributed to the study design, supervision of the molecular biology experiments, data analyses and interpretation, and has written the manuscript.

All authors have approved the final version of the manuscript. 


\section{Literature Cited}

Almendros I, Carreras A, Ramirez J, Montserrat JM, Navajas D, Farre R (2008). Upper airway collapse and reopening induce inflammation in a sleep apnoea model. Eur Respir $\mathrm{J}$ 32:399-404.

Altay T, Gonzales ER, Park TS, Gidday JM (2004). Cerebrovascular inflammation after brief episodic hypoxia: modulation by neuronal and endothelial nitric oxide synthase. J Appl Physiol (1985 ) 96:1223-1230.

Barreiro E, Bustamante V, Cejudo P, Galdiz JB, Gea J, de LP, Martinez-Llorens J, Ortega F, Puente-Maestu L, Roca J, Rodriguez-Gonzalez Moro JM (2015). Guidelines for the evaluation and treatment of muscle dysfunction in patients with chronic obstructive pulmonary disease. Arch Bronconeumol 51:384-395.

Barreiro E, Galdiz JB, Marinan M, Alvarez FJ, Hussain SN, Gea J (2006). Respiratory loading intensity and diaphragm oxidative stress: N-acetyl-cysteine effects. J Appl Physiol 100:555-563.

Barreiro E, Nowinski A, Gea J, Sliwinski P (2007). Oxidative stress in the external intercostal muscles of patients with obstructive sleep apnoea. Thorax 62:1095-1101.

Barreiro E, Peinado VI, Galdiz JB, Ferrer E, Marin-Corral J, Sanchez F, Gea J, Barbera JA (2010). Cigarette smoke-induced oxidative stress: A role in chronic obstructive pulmonary disease skeletal muscle dysfunction. Am J Respir Crit Care Med 182:477-488.

Barreiro E, Sanchez D, Galdiz JB, Hussain SN, Gea J (2005). N-acetylcysteine increases manganese superoxide dismutase activity in septic rat diaphragms. Eur Respir J 26:10321039.

Barreiro E, Schols AM, Polkey MI, Galdiz JB, Gosker HR, Swallow EB, Coronell C, Gea J (2008). Cytokine profile in quadriceps muscles of patients with severe COPD. Thorax 63:100107.

Bigard AX, Brunet A, Guezennec CY, Monod H (1991). Skeletal muscle changes after endurance training at high altitude. J Appl Physiol (1985 ) 71:2114-2121.

Chacon-Cabrera A, Fermoselle C, Salmela I, Yelamos J, Barreiro E (2015). MicroRNA expression and protein acetylation pattern in respiratory and limb muscles of Parp-1(-/-) and Parp-2(-/-) mice with lung cancer cachexia. Biochim Biophys Acta 1850:2530-2543.

Chacon-Cabrera A, Fermoselle C, Urtreger AJ, Mateu-Jimenez M, Diament MJ, de Kier Joffe ED, Sandri M, Barreiro E (2014a). Pharmacological strategies in lung cancer-induced cachexia: effects on muscle proteolysis, autophagy, structure, and weakness. J Cell Physiol 229:1660-1672.

Chacon-Cabrera A, Rojas Y, Martinez-Caro L, Vila-Ubach M, Nin N, Ferruelo A, Esteban A, Lorente JA, Barreiro E (2014b). Influence of mechanical ventilation and sepsis on redox balance in diaphragm, myocardium, limb muscles, and lungs. Transl Res 164:477-495.

Chen L, Einbinder E, Zhang Q, Hasday J, Balke CW, Scharf SM (2005). Oxidative stress and left ventricular function with chronic intermittent hypoxia in rats. Am J Respir Crit Care Med 172:915-920. 
de Senzi Moraes PR, Ferretti R, Moraes LH, Neto HS, Marques MJ, Minatel E (2013). Nacetylcysteine treatment reduces TNF-alpha levels and myonecrosis in diaphragm muscle of mdx mice. Clin Nutr 32:472-475.

de Theije CC, Langen RC, Lamers WH, Gosker HR, Schols AM, Kohler SE (2015). Differential sensitivity of oxidative and glycolytic muscles to hypoxia-induced muscle atrophy. J Appl Physiol (1985 ) 118:200-211.

Dominguez-Alvarez M, Sabate-Bresco M, Vila-Ubach M, Galdiz JB, Alvarez FJ, Casadevall C, Gea J, Barreiro E (2014). Molecular and physiological events in respiratory muscles and blood of rats exposed to inspiratory threshold loading. Transl Res 163:478-493.

Dunleavy M, Dooley M, Cox D, Bradford A (2005). Chronic intermittent asphyxia increases platelet reactivity in rats. Exp Physiol 90:411-416.

Eckert DJ, Malhotra A, Jordan AS (2009). Mechanisms of apnea. Prog Cardiovasc Dis 51:313-323.

Fermoselle C, Rabinovich R, Ausin P, Puig-Vilanova E, Coronell C, Sanchez F, Roca J, Gea J, Barreiro E (2012). Does oxidative stress modulate limb muscle atrophy in severe COPD patients? Eur Respir J 40:851-862.

Garcia-Talavera I, Jimenez GP, Dorta SR (2015). Exercise-induced oxygen desaturation in chronic obstructive pulmonary disease patients. Arch Bronconeumol 51:481-482.

Garvey JF, Taylor CT, McNicholas WT (2009). Cardiovascular disease in obstructive sleep apnoea syndrome: the role of intermittent hypoxia and inflammation. Eur Respir J 33:11951205.

Gautier-Veyret E, Arnaud C, Back M, Pepin JL, Petri MH, Baguet JP, Tamisier R, Levy P, Stanke-Labesque F (2013). Intermittent hypoxia-activated cyclooxygenase pathway: role in atherosclerosis. Eur Respir J 42:404-413.

Giordano C, Lemaire C, Li T, Kimoff RJ, Petrof BJ (2015). Autophagy-associated atrophy and metabolic remodeling of the mouse diaphragm after short-term intermittent hypoxia. PLoS One 10:e131068.

Grounds MD, Torrisi J (2004). Anti-TNFalpha (Remicade) therapy protects dystrophic skeletal muscle from necrosis. FASEB J 18:676-682.

Howald H, Pette D, Simoneau JA, Uber A, Hoppeler H, Cerretelli P (1990). Effect of chronic hypoxia on muscle enzyme activities. Int J Sports Med 11 Suppl 1:S10-S14.

Koechlin C, Maltais F, Saey D, Michaud A, LeBlanc P, Hayot M, Prefaut C (2005). Hypoxaemia enhances peripheral muscle oxidative stress in chronic obstructive pulmonary disease. Thorax 60:834-841.

Kwast KE, Hand SC (1996). Acute depression of mitochondrial protein synthesis during anoxia: contributions of oxygen sensing, matrix acidification, and redox state. J Biol Chem 271:7313-7319.

Langen RC, Schols AM, Kelders MC, Van Der Velden JL, Wouters EF, Janssen-Heininger YM (2002). Tumor necrosis factor-alpha inhibits myogenesis through redox-dependent and independent pathways. Am J Physiol Cell Physiol 283:C714-C721. 
Lavie L (2008). Intermittent hypoxia: the culprit of oxidative stress, vascular inflammation and dyslipidemia in obstructive sleep apnea. Expert Rev Respir Med 2:75-84.

Lavie L (2012). Oxidative stress inflammation and endothelial dysfunction in obstructive sleep apnea. Front Biosci (Elite Ed) 4:1391-1403.

Lavie L, Dyugovskaya L, Golan-Shany O, Lavie P (2010). Heat-shock protein 70: expression in monocytes of patients with sleep apnoea and association with oxidative stress and tumour necrosis factor-alpha. J Sleep Res 19:139-147.

Lin CP, Huang PH, Lai CF, Chen JW, Lin SJ, Chen JS (2015). Simvastatin Attenuates Oxidative Stress, NF-kappaB Activation, and Artery Calcification in LDLR-/- Mice Fed with High Fat Diet via Down-regulation of Tumor Necrosis Factor-alpha and TNF Receptor 1. PLoS One 10:e143686.

Ling Q, Sailan W, Ran J, Zhi S, Cen L, Yang X, Xiaoqun Q (2008). The effect of intermittent hypoxia on bodyweight, serum glucose and cholesterol in obesity mice. Pak J Biol Sci 11:869-875.

Maltais F, Decramer M, Casaburi R, Barreiro E, Burelle Y, Debigare R, Dekhuijzen PN, Franssen F, Gayan-Ramirez G, Gea J, Gosker HR, Gosselink R, Hayot M, Hussain SN, Janssens W, Polkey MI, Roca J, Saey D, Schols AM, Spruit MA, Steiner M, Taivassalo T, Troosters T, Vogiatzis I, Wagner PD (2014). An official American Thoracic Society/European Respiratory Society statement: update on limb muscle dysfunction in chronic obstructive pulmonary disease. Am J Respir Crit Care Med 189:e15-e62.

Marin-Corral J, Minguella J, Ramirez-Sarmiento AL, Hussain SN, Gea J, Barreiro E (2009). Oxidised proteins and superoxide anion production in the diaphragm of severe COPD patients. Eur Respir J 33:1309-1319.

Martinez CE, Casitas MR, Garcia-Rio F (2015). Sleep apnea-hypopnea syndrome and type 2 diabetes. A reciprocal relationship? Arch Bronconeumol 51:128-139.

Martinez-Garcia MA, Campos-Rodriguez F, Almendros I, Farre R (2015). Relationship Between Sleep Apnea and Cancer. Arch Bronconeumol 51:456-461.

McGuire M, MacDermott M, Bradford A (2003). Effects of chronic intermittent asphyxia on rat diaphragm and limb muscle contractility. Chest 123:875-881.

Moraes LH, Bollineli RC, Mizobuti DS, Silveira LR, Marques MJ, Minatel E (2015). Effect of $\mathrm{N}$-acetylcysteine plus deferoxamine on oxidative stress and inflammation in dystrophic muscle cells. Redox Rep 20:109-115.

Ordas I, Mould DR, Feagan BG, Sandborn WJ (2012). Anti-TNF monoclonal antibodies in inflammatory bowel disease: pharmacokinetics-based dosing paradigms. Clin Pharmacol Ther 91:635-646.

Preedy VR, Smith DM, Sugden PH (1985). The effects of 6 hours of hypoxia on protein synthesis in rat tissues in vivo and in vitro. Biochem J 228:179-185.

Puig-Vilanova E, Martinez-Llorens J, Ausin P, Roca J, Gea J, Barreiro E (2015a). Quadriceps muscle weakness and atrophy are associated with a differential epigenetic profile in advanced COPD. Clin Sci (Lond) 128:905-921. 
Puig-Vilanova E, Rodriguez DA, Lloreta J, Ausin P, Pascual-Guardia S, Broquetas J, Roca J, Gea J, Barreiro E (2015b). Oxidative stress, redox signaling pathways, and autophagy in cachectic muscles of male patients with advanced COPD and lung cancer. Free Radic Biol Med 79:91-108.

Shortt CM, Fredsted A, Bradford A, O'Halloran KD (2013). Diaphragm muscle remodeling in a rat model of chronic intermittent hypoxia. J Histochem Cytochem 61:487-499.

Shortt CM, Fredsted A, Chow HB, Williams R, Skelly JR, Edge D, Bradford A, O'Halloran $\mathrm{KD}$ (2014). Reactive oxygen species mediated diaphragm fatigue in a rat model of chronic intermittent hypoxia. Exp Physiol 99:688-700.

Sigala I, Zacharatos P, Boulia S, Toumpanakis D, Michailidou T, Parthenis D, Roussos C, Papapetropoulos A, Hussain SN, Vassilakopoulos T (2012). Nitric oxide regulates cytokine induction in the diaphragm in response to inspiratory resistive breathing. J Appl Physiol (1985 ) 113:1594-1603.

Sigala I, Zacharatos P, Toumpanakis D, Michailidou T, Noussia O, Theocharis S, Roussos C, Papapetropoulos A, Vassilakopoulos T (2011). MAPKs and NF-kappaB differentially regulate cytokine expression in the diaphragm in response to resistive breathing: the role of oxidative stress. Am J Physiol Regul Integr Comp Physiol 300:R1152-R1162.

Slot IG, Schols AM, de Theije CC, Snepvangers FJ, Gosker HR (2016). Alterations in Skeletal Muscle Oxidative Phenotype in Mice Exposed to 3 Weeks of Normobaric Hypoxia. J Cell Physiol 231:377-392.

Taivassalo T, Hussain SN (2016). Contribution of the Mitochondria to Locomotor Muscle Dysfunction in Patients With COPD. Chest 149:1302-1312.

Toumpanakis D, Karatza MH, Katsaounou P, Roussos C, Zakynthinos S, Papapetropoulos A, Vassilakopoulos T (2009). Antioxidant supplementation alters cytokine production from monocytes. J Interferon Cytokine Res 29:741-748.

Troncoso Brindeiro CM, da Silva AQ, Allahdadi KJ, Youngblood V, Kanagy NL (2007). Reactive oxygen species contribute to sleep apnea-induced hypertension in rats. Am J Physiol Heart Circ Physiol 293:H2971-H2976.

Vassilakopoulos T, Divangahi M, Rallis G, Kishta O, Petrof B, Comtois A, Hussain SN (2004). Differential cytokine gene expression in the diaphragm in response to strenuous resistive breathing. Am J Respir Crit Care Med 170:154-161.

Vassilakopoulos T, Karatza MH, Katsaounou P, Kollintza A, Zakynthinos S, Roussos C (2003). Antioxidants attenuate the plasma cytokine response to exercise in humans. J Appl Physiol (1985 ) 94:1025-1032.

Vassilakopoulos T, Katsaounou P, Karatza MH, Kollintza A, Zakynthinos S, Roussos C (2002). Strenuous resistive breathing induces plasma cytokines: role of antioxidants and monocytes. Am J Respir Crit Care Med 166:1572-1578. 


\section{FIGURE LEGENDS}

Figure 1: Schematic representation of mean values of body weights in all study groups of rats (non-exposed control, hypoxia, anti-TNF-alpha-hypoxia, NAC-hypoxia) on days 0, 7 and 14 .

Figure 2: Mean values and standard deviation of TNF-alpha protein levels measured in the diaphragm, gastrocnemius, and plasma compartments (top, medium, and bottom panels, respectively, $\mathrm{N}=8$ / group all groups). In the diaphragm, protein levels of TNF-alpha were significantly increased in rats exposed to hypoxia compared to the non-exposed controls $(* * *$, $\mathrm{p}<0.001)$. Additionally, the respiratory muscle of rats treated with anti-TNF-alpha antibody exhibited a larger increase $(\mathbb{\uparrow}, \mathrm{p}<0.05)$ in TNF-alpha protein levels compared to rats exposed to hypoxia-only and non-exposed controls $(*, \mathrm{p}<0.05)$, while a significant decrease was observed in animals treated with NAC $(\boldsymbol{\Phi} \uparrow, \mathrm{p}<0.01)$. In the limb muscles no significant differences were found in any of the groups (n.s., non-significant). In plasma, TNF-alpha was significantly greater $(*, p<0.05)$ in rats exposed to hypoxia, while no differences (n.s., nonsignificant were seen in the group of hypoxia anti-TNF-alpha rats compared to the nonexposed controls, and a significant decline was observed in animals treated with NAC compared to hypoxia only rats $(\mathbb{\Phi}, \mathrm{p}<0.05)$.

Figure 3: Mean values and standard deviation of IL-1beta protein levels measured in the diaphragm, gastrocnemius, and plasma compartments (top, medium, and bottom panels, respectively, $\mathrm{N}=8$ / group all groups). Protein levels of IL-1beta were significantly increased $(* *, p<0.01)$ in the diaphragm of hypoxia rats compared to the non-exposed controls. Moreover, protein levels of this cytokine were even greater $(\mathbb{\Phi}, \mathrm{p}<0.05)$ in the diaphragms of rats treated with anti-TNF-alpha antibody than in hypoxia-only rats and the non-exposed controls $(*, p<0.05)$. A significant decrease was observed in animals treated with NAC compared to hypoxia-only group $(\mathbb{\Phi}, \mathrm{p}<0.05)$, although these were significantly greater compared to controls rodents. No significant differences were observed among any of the study groups in IL-1beta protein levels in the limb muscle (n.s., non-significant). In plasma, 
IL-1beta was significantly greater $(*, \mathrm{p}<0.05)$ in rats exposed to hypoxia than in the nonexposed controls, while a significant decrease was observed in animals treated with either anti-TNF-alpha antibody or NAC $(\boldsymbol{\Phi}, \mathrm{p}<0.05)$.

Figura 4: Mean values and standard deviation of IL-6 protein levels measured in the diaphragm, gastrocnemius, and plasma compartments (top, medium, and bottom panels, respectively, $\mathrm{N}=8$ / group all groups). In the respiratory muscle, IL-6 levels were significantly greater $(* *, p<0.01)$ in hypoxia rats compared to the non-exposed controls. Additionally, a greater increase $(\boldsymbol{\Phi}, \mathrm{p}<0.05)$ in IL-6 protein levels was observed in the diaphragms of rats treated with anti-TNF-alpha antibody compared to rats exposed to hypoxia and the nonexposed controls $(* * *, \mathrm{p}<0.001)$. Concomitant treatment with NAC did not elicit any significant difference in IL-6 levels compared to hypoxia rats, although IL-6 levels were significantly higher than in non-exposed control rats No significant differences were observed among any of the estudy groups in IL-6 protein levels in either limb muscles or plasma (n.s., non significant).

Figure 5: Mean values and standard deviation of interferon-gamma protein levels measured in the diaphragm, gastrocnemius, and plasma compartments (top, medium, and bottom panels, respectively, $\mathrm{N}=8$ / group all groups). $6 \mathrm{~A}$ ) In the respiratory muscle, interferon-gamma levels were significantly greater in hypoxia rats compared to the non-exposed controls $(*, p<0.05)$. Moreover, protein levels of this cytokine were even greater $(\boldsymbol{q}, \mathrm{p}<0.05)$ in the diaphragm of rats treated with anti-TNF-alpha antibody compared to hypoxia-only rats and the non-exposed controls $(* *, \mathrm{p}<0.01)$. Concomitant treatment with NAC did not elicit any significant difference in interferon-gamma levels compared to hypoxia-only rats. No significant differences were observed among any of the study groups in interferon-gamma protein levels in either limb muscles or plasma (n.s., non significant).

Figure 6: Mean values and standard deviation of superoxide anion levels measured in the diaphragm, gastrocnemius, and plasma compartments (Figure 6A, Figure 6B, Figure 6C, 
respectively, $\mathrm{N}=8$ / group all groups). 6A) In both mitochondrial and membrane diaphragm muscle compartments, levels of superoxide anion were significantly increased in the hypoxia group of rats compared to the non-exposed controls $(*, p<0.05)$, while a significant decrease was observed in the treated groups with either anti-TNF-alpha antibody or NAC in both muscle fractions ( $\mathbb{\uparrow}, \mathrm{p}<0.05$ for all groups, except for the membrane compartment treated with anti-TNF-alpha antibody: $\uparrow \uparrow, p<0.01)$. No significant differences were observed in superoxide anion levels produced within the cytosol compartment among any of the study groups (n.s., non-significant). 6B) In the gastrocnemius, levels of superoxide anion were significantly increased in the mitochondrial compartment of the hypoxia group of rats compared to the non-exposed controls $(*, \mathrm{p}<0.05)$, while concomitant treatment with either anti-TNF-alpha antibody or NAC induced a significant decline in superoxide anion levels compared to hypoxia only-rodents ( $(\uparrow \uparrow \uparrow, \mathrm{p}<0.001$; and $\uparrow, \mathrm{p}<0.05$, respectively). No significant differences were observed among any of the study groups in superoxide anion levels in either membrane or cytosolic compartments (n.s., non-significant). 6C) In plasma, levels of superoxide anion were significantly increased in the hypoxia group of rats compared to the non-exposed controls $(*, \mathrm{p}<0.05)$, while concomitant treatment with anti-TNF-alpha antibody or NAC induced a significant decrease in superoxide anion levels compared to hypoxia-only rats ( $\mathbf{q}$, $\mathrm{p}<0.01$; and $\uparrow, \mathrm{p}<0.05$, respectively). 
1

2

3

4

5

6

7

8

9

10

11

12

13

14

15

16

17

18

19

20

21

22

23

24

25

26

27

28

29

30

31

32

33

34

35

36

37

38

39

40

41

42

43

44

45

46

47

48

49

50

51

52

53

54

55

56

57

58

59

60

Table 1. Body weight changes in the different study groups of animals at baseline and at the end of the study period (14 days).

\begin{tabular}{|c|c|c|c|c|}
\hline & $\begin{array}{c}\text { Non- } \\
\text { exposed } \\
\text { controls }\end{array}$ & Hypoxia & $\begin{array}{c}\text { Hypoxia-anti-TNF- } \\
\text { alpha }\end{array}$ & Hypoxia-NAC \\
\hline $\begin{array}{c}\text { Initial body weight, g } \\
\text { (day 0) }\end{array}$ & $252(5)$ & $276(12)$ & $263(9)$ & $292(5)$ \\
\hline $\begin{array}{c}\text { Final body weight, g } \\
\text { (day 14) }\end{array}$ & $279(7) \dagger \dagger \dagger$ & $272(10)$, n.s. & $261(7)$, n.s. & $290(26)$, n.s. \\
\hline $\begin{array}{c}\text { Percentage of } \\
\text { body weight gain }\end{array}$ & $\mathbf{+ 9 . 8 0 \%}$ & $\mathbf{- 1 . 5 0 \% * * *}$ & $\mathbf{- 0 . 8 0 \% * * *}$ & $\mathbf{- 1 . 5 1 \% * *}$ \\
\hline
\end{tabular}

Values are expressed as mean (standard desviation).

Definition of abbreviations: NAC, N-acetyl cysteine; TNF, tumor necrosis factor; g, grams.

Statistical significance: n.s.: non significant; $\dagger \dagger \dagger \mathrm{p}<0.001$ between day 14 and baseline time-points; ** $\mathrm{p}<$ 0.01 and $* * * \mathrm{p}<0.001$ between any of the hypoxia experimental groups of rats and the non-exposed control animals. 
Table 2. Fiber type composition in the diaphragm and gastrocnemius muscles of all study groups of rats at the end of the study period (day 14)

\begin{tabular}{|c|c|c|c|c|c|}
\hline & & $\begin{array}{c}\text { Non- } \\
\text { exposed } \\
\text { controls }\end{array}$ & Hypoxia & $\begin{array}{l}\text { Hypoxia-anti-TNF- } \\
\text { alpha }\end{array}$ & Hypoxia-NAC \\
\hline \multirow{2}{*}{ Type I fibers (\%) } & Diaphragm & $33(5)$ & $31(7)$, n.s. & 32 (4), n.s. & $33(7)$, n.s. \\
\hline & Gastrocnemius & $25(6)$ & 23 (6), n.s. & 23 (4), n.s. & 20 (4), n.s. \\
\hline \multirow{2}{*}{ Type II fibers (\%) } & Diaphragm & $67(5)$ & 69 (7), n.s. & 68 (4), n.s. & 67 (7), n.s. \\
\hline & Gastrocnemius & $75(6)$ & 77 (7), n.s. & 77 (4), n.s. & 80 (4), n.s. \\
\hline \multirow{2}{*}{$\begin{array}{l}\text { Cross-sectional area } \\
\text { type I fibers }\left(\mu \mathbf{m}^{2}\right)\end{array}$} & Diaphragm & $678(148)$ & 585 (91), n.s. & 669 (104), n.s. & 635 (132), n.s. \\
\hline & Gastrocnemius & $1,173(338)$ & 1,132 (440), n.s. & 1,042 (124), n.s. & 917 (180), n.s. \\
\hline \multirow{2}{*}{$\begin{array}{l}\text { Cross-sectional area } \\
\text { type II fibers }\left(\mu \mathbf{m}^{2}\right)\end{array}$} & Diaphragm & $823(190)$ & 837 (130), n.s. & 838 (112), n.s. & 834 (167), n.s. \\
\hline & Gastrocnemius & $1,381(281)$ & 1,392 (172), n.s. & $1,211(81)$, n.s. & 1,235 (48), n.s. \\
\hline
\end{tabular}

Values are expressed as mean (standard desviation).

Definition of abbreviations: NAC, N-acetyl cysteine; TNF, tumor necrosis factor

Statistical significance: n.s.: non significant. 
Table 3. Structural abnormalities in the diaphragm and gastrocnemius muscles of all study groups of rats at the end of the study period (day 14)

\begin{tabular}{|c|c|c|c|c|c|}
\hline & & $\begin{array}{c}\text { Non-exposed } \\
\text { controls }\end{array}$ & Hypoxia & $\begin{array}{c}\text { Hypoxia -anti-TNF- } \\
\text { alpha } \\
\end{array}$ & Hypoxia-NAC \\
\hline \multirow{2}{*}{$\begin{array}{c}\text { Total } \\
\text { abnormalities (\%) }\end{array}$} & Diaphragm & $4.02(1.3) \%$ & $5.49(0.9) \% *$ & $12.21(2.6) \%$ IIIII,**** & 10.55 (1.8)\% IIIII \\
\hline & Gastrocnemius & $3.6(0.5) \%$ & $6.18(1.6) \% *$ & $9.11(0.5) \%$ II,*** & $8.16(0.4) \%$ II, ** \\
\hline \multirow{2}{*}{ Internal nuclei } & Diaphragm & $36.37(12)$ & $62.8(25) *$ & 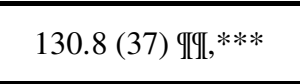 & 129.5 (25) बIIII,**** \\
\hline & Gastrocnemius & $26(3)$ & $53.5(19) *$ & $81(30) \mathbb{d}[, * *$ & 72.25 (16) II,** \\
\hline \multirow{2}{*}{$\begin{array}{l}\text { Inflammatory } \\
\text { cells }\end{array}$} & Diaphragm & $40(31)$ & $70.6(21) *$ & 144 (30) IIIf;*** & 103.25 (24) II,**** \\
\hline & Gastrocnemius & $55(11)$ & $77.5(26) *$ & $102.25(15) \mathrm{gI}, * *$ & 104.2 (11) gI,** \\
\hline
\end{tabular}

Values are expressed as mean (standard desviation).

Definition of abbreviations: NAC: N-acetyl cysteine; TNF, tumor necrosis factor.

Values in the different categories are expressed as absolute values of the total amount of each item counted in the analyzed fields $(1,890)$ of the muscles. However, total abnormalities are expressed as the percentage of items considered to be abnormal within the total number of fields (1890, normal and abnormal) examined in the muscles of all study animals.

Statistical significance: n.s.: non-significant $* \mathrm{p}:<0.05$ between any of the hypoxia groups and the nonexposed control animals, and II $\mathrm{p}:<0.05$, III $\mathrm{p}:<0.01$, IITI $\mathrm{p}:<0.001$ between any of the pharmacologically treated groups exposed to hypoxia and hypoxia-only rats. 


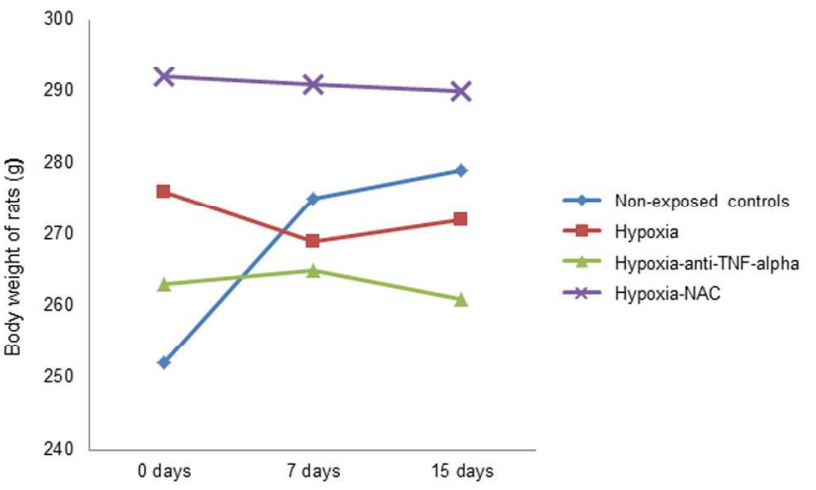

254x190mm (300 x 300 DPI)

John Wiley \& Sons, Inc. 
Dominguez-Alvarez et al. Figure 2
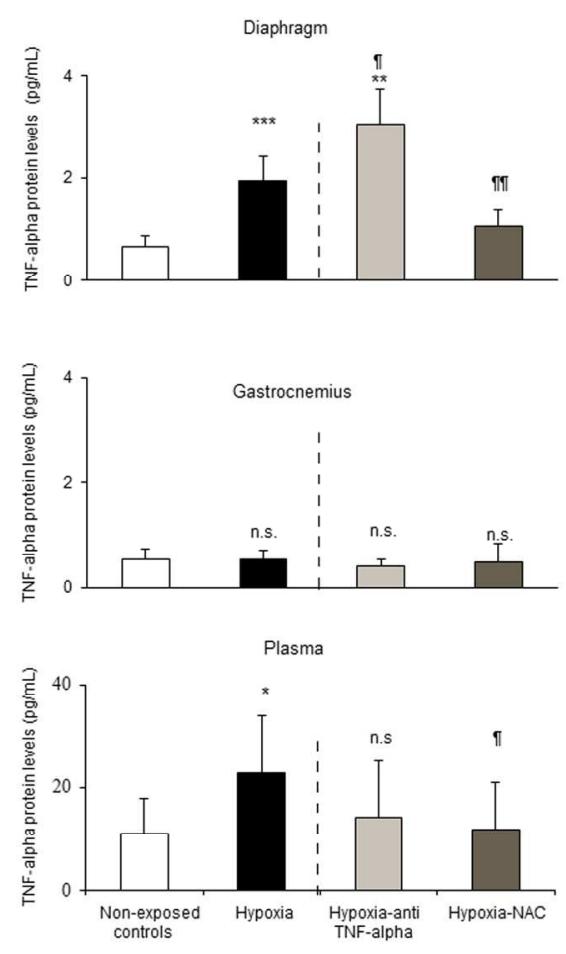

$254 \times 190 \mathrm{~mm}(300 \times 300 \mathrm{DPI})$

John Wiley \& Sons, Inc. 
Domínguez-Álvarez et al. Figure 3
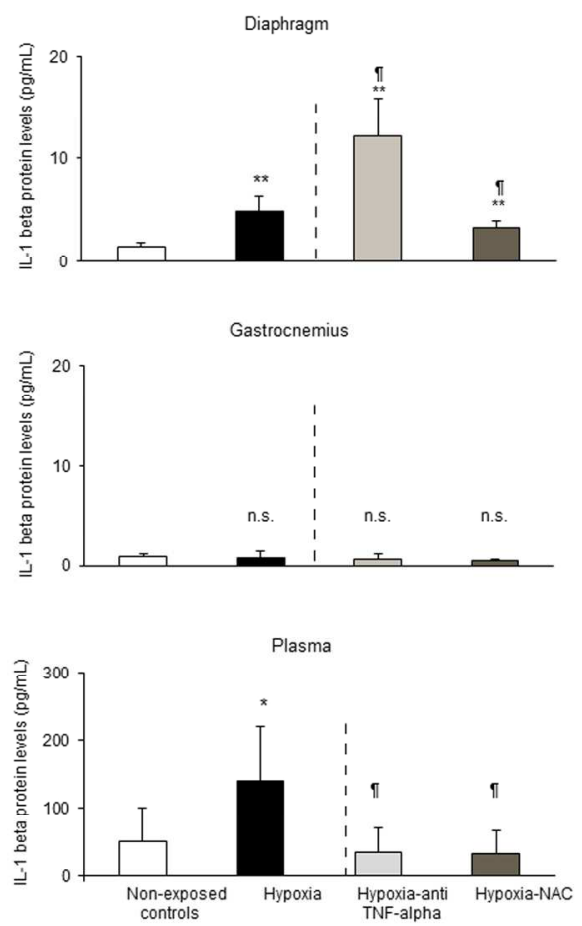

$254 \times 190 \mathrm{~mm}(300 \times 300 \mathrm{DPI})$

John Wiley \& Sons, Inc. 


1
2
3
4
5
6
7
8
9
10
11
12
13
14
15
16
17
18
19
20
21
22
23
24
25
26
27
28
29
30
31
32
33
34
35
36
37
38
39
40
41
42
43
40
45
49
50
51
52
53
55
50

Dominguez-Âlvarez et al. Figure 4
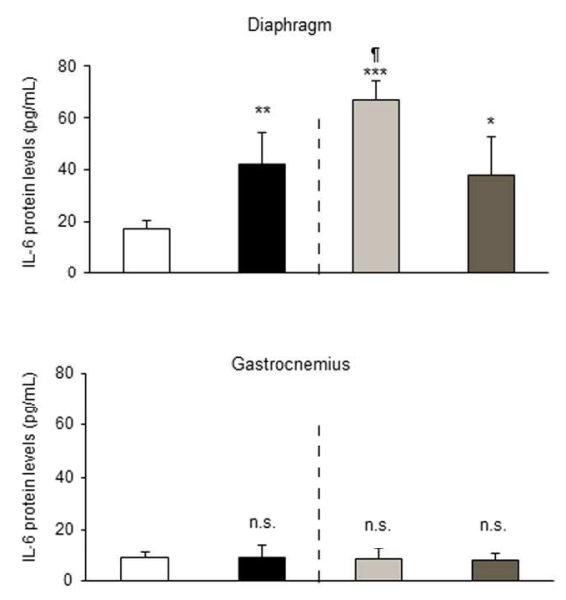

Plasma

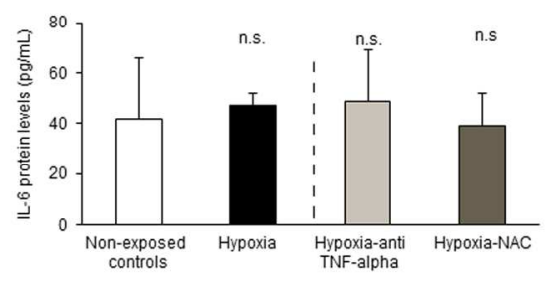

$254 \times 190 \mathrm{~mm}(300 \times 300 \mathrm{DPI})$

John Wiley \& Sons, Inc. 
Dominguez-Âlvarez et al. Figure 5
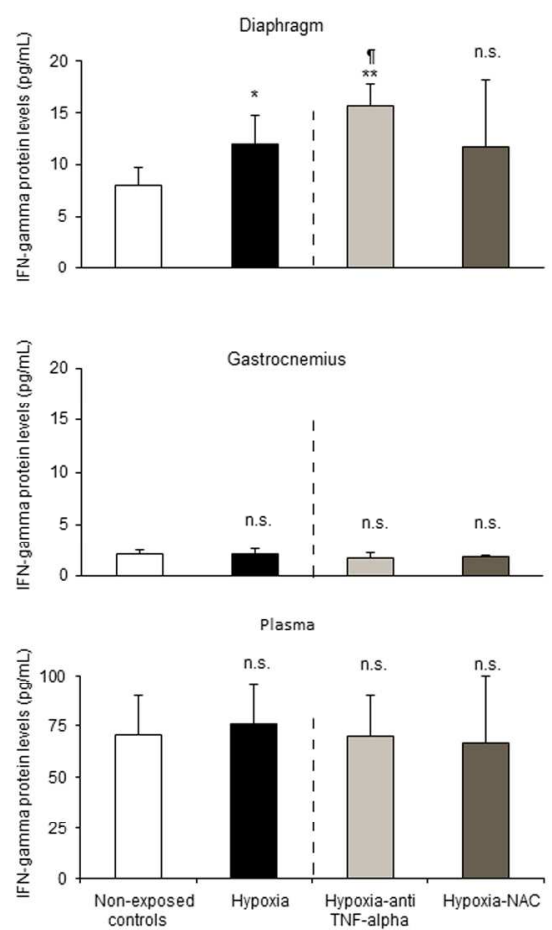

$254 \times 190 \mathrm{~mm}(300 \times 300$ DPI $)$

John Wiley \& Sons, Inc. 
Diaphragm

Mitochondria
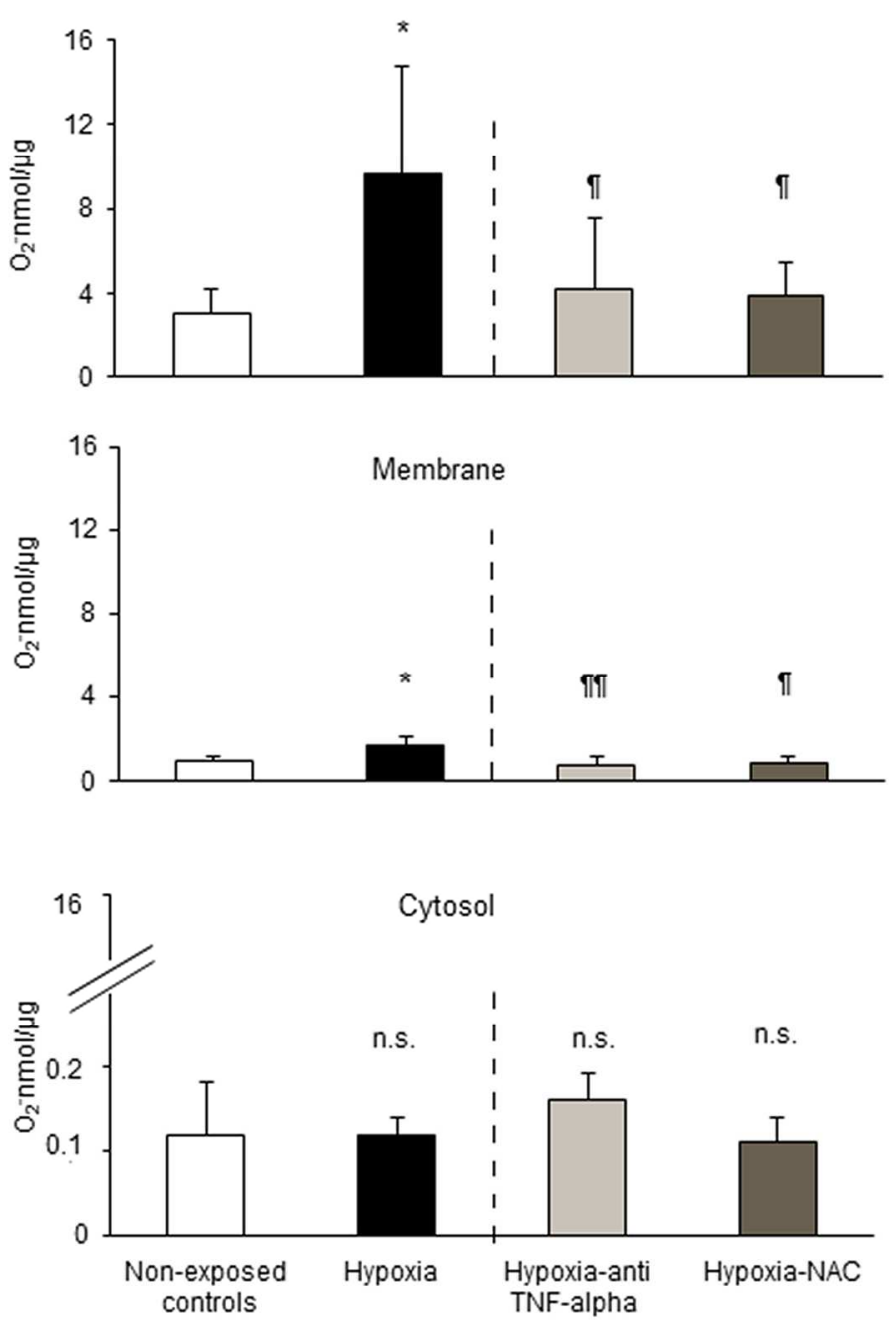

$1168 \times 1807 \mathrm{~mm}(96 \times 96 \mathrm{DPI})$

John Wiley \& Sons, Inc. 


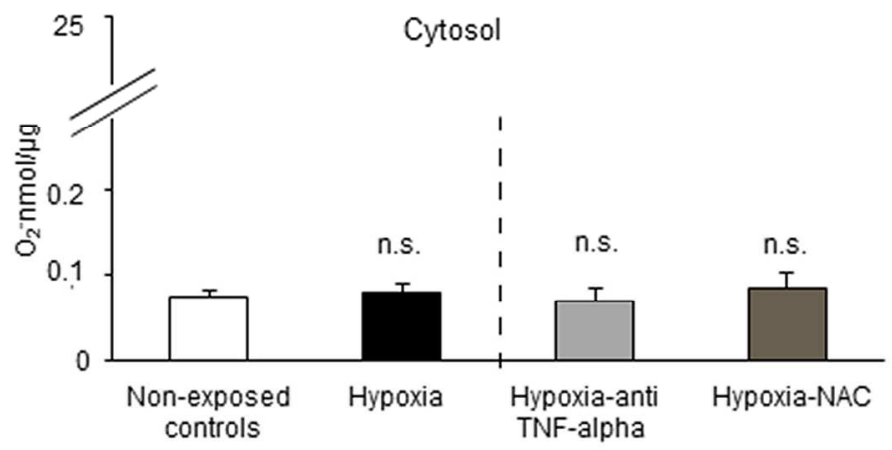

1096x1790mm (96 x 96 DPI)

John Wiley \& Sons, Inc. 
1

2

3

4

5

6

7

8

9

10

11

12

13

14

15

16

17

18

19

20

21

22

23

24

25

26

27

28

29

30

31

32

33

34

35

36

37

38

39

40

41

42

43

44

45

46

47

48

49

50

51

52

53

54

55

56

57

58

59

60
Dominguez-Álvarez et al. Figure $6 \mathrm{C}$

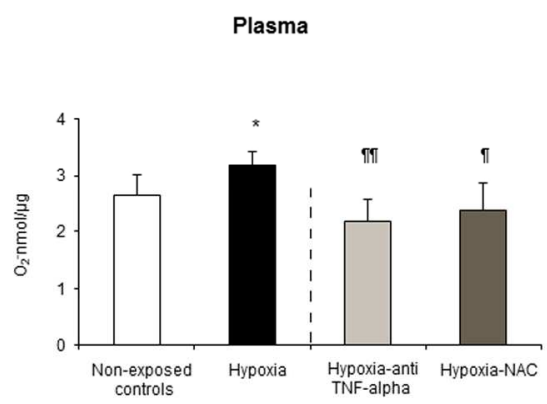

$254 \times 190 \mathrm{~mm}(300 \times 300$ DPI $)$

John Wiley \& Sons, Inc. 